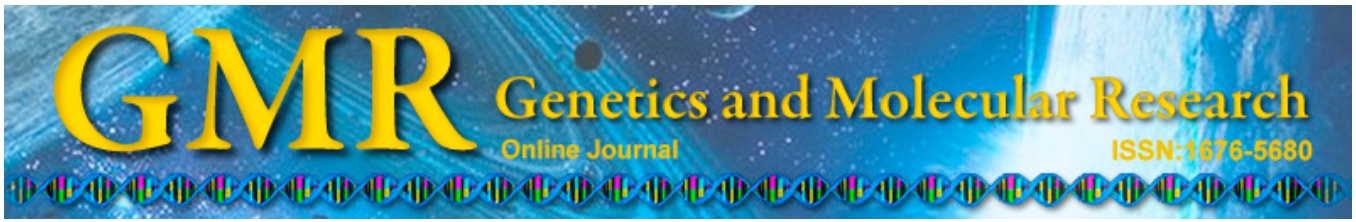

\title{
Influence of sugars and hormones on the genes involved in sucrose metabolism in maize endosperms
}

\author{
X.D. Ren ${ }^{1,2}$, H.M. Liu', Y.H. Liu' ${ }^{2}$, Y.F. Hu ${ }^{3}$, J.J. Zhang ${ }^{1,2}$ and Y.B. Huang ${ }^{2,3}$ \\ ${ }^{1}$ College of Life Science, Sichuan Agricultural University, Ya'an, China \\ ${ }^{2}$ Maize Research Institute, Sichuan Agricultural University, Chengdu, China \\ ${ }^{3}$ College of Agronomy, Sichuan Agricultural University, Chengdu, China \\ Corresponding authors: J.J. Zhang / Y.B. Huang \\ E-mail: junjiezh@163.com / yubihuang@sina.com
}

Genet. Mol. Res. 14 (1): 1671-1678 (2015)

Received April 7, 2013

Accepted August 8, 2014

Published March 6, 2015

DOI http://dx.doi.org/10.4238/2015.March.6.13

\begin{abstract}
Starch is the major storage product in the endosperm of cereals. Its synthesis is closely related to sucrose metabolism. In our previous study, we found that the expression of most of the genes involved in starch synthesis might be regulated by sugars and hormones in the maize endosperm. However, little is known regarding the transcriptional regulation of genes involved in sucrose metabolism. Thus, in this study, maize endosperms were treated with different sugars and hormones and the expression of genes involved in sucrose metabolism (including synthesis, degradation, and transport) were evaluated using real-time quantitative reverse transcription-polymerase chain reaction. We found that genes affected by different sugars and hormones were primarily regulated by abscisic acid. Sucrose and abscisic acid showed an additive effect on the expression of some genes. Differences in the transcriptional
\end{abstract}


regulation of genes involved in sucrose metabolism and starch biosynthesis were observed.

Key words: Endosperm; Gene expression; Hormone; Maize; Sugar

\section{INTRODUCTION}

Photosynthesis converts energy from the sun into chemical energy (sugars) in green plants. Sucrose is the main form of sugar subjected to long-distance transport and distribution in higher plants. After synthesis in autotrophic organs (source), sucrose is loaded into the sieve element/companion cell complex and transported into heterotrophic organs (sink), primarily for synthesis of storage molecules or to provide energy for the plant itself. In cereal crops, except for a small amount sucrose that is utilized during metabolism, most sucrose in the sink organs is converted into starch. In addition, sucrose is involved in abiotic stress tolerance in plants (Gupta and Kaur, 2005; Cui et al., 2010). Importantly, sucrose is an important signaling molecule in plants that controls the transcription and translation of a number of genes (Shin et al., 2013; Tognetti et al., 2013). The main metabolic pathways of sucrose are well-known. Three enzymes and 1 protein participate in sucrose synthesis, degradation, and transport, including invertase (Inv), sucrose synthase, sucrose-phosphate synthase (SPS), and sucrose transporters/sucrose carriers (SUTs/SUCs). Inv and sucrose synthase are mainly involved in sucrose degradation, while SPS is responsible for sucrose synthesis; the transport of sucrose from source to sink organs is performed by SUTs.

The genes encoding these enzymes and protein have been cloned from many plants, and various studies have examined their expression and regulation, structure and function, and related enzymatic properties (Ren and Zhang, 2013). In maize, 6 genes encoding Inv have been identified, including Ivr 1, Ivr2, Incw1, Incw2 (Mn1), Incw3, and Incw4 (Shanker et al., 1995; Xu et al., 1995, 1996; Cheng et al., 1996; Kim et al., 2000b). The expression of $I v r l$ and $I v r 2$ is regulated by sugars and oxygen conditions (Xu et al., 1996; Zeng et al., 1999). In addition, $I v r 2$ was found to be specifically induced under water stress (Kim et al., 2000a; Trouverie et al., 2003). Incw 1 and Incw2 expression showed significant spatial and temporal heterogeneity in the developing endosperm (Chourey et al., 2006). Three genes encode maize sucrose synthase, including Sh1, Sus 1, and Sus2 (Werr et al., 1985; Gupta et al., 1988; Carlson et al., 2002). Expression of these genes shows tissue/organ and developmental specificity. Shl and Sus 1 transcript levels are regulated by glucose, and treatment with different glucose concentration impact expression levels (Koch et al., 1992). Several studies found Sh1 and Sus 1 to be modulated by oxygen conditions at the transcriptional and translational level (Mcelfresh and Chourey, 1988; Rowland et al., 1989; Zeng et al., 1998). At least 7 sucrose-phosphate synthase genes have been identified in the maize genome to date, known as ZmSPS1-ZmSPS7. Cold stress can induce ZmSPS6 transcription and inhibit $Z m S P S 2,4$, and 7 transcriptions. Drought can induce ZmSPS3 expression and inhibit $\mathrm{Zm}$ $S P S 1,4$, and 6 expressions. Under low nitrogen conditions, ZmSPS2 expression was inhibited, while ZmSPS3 and 6 were induced (Lutfiyya et al., 2007). Seven genes encoding SUTs have been identified in maize, known as ZmSUT1-ZmSUT6 and ZmERD6 (Ma et al., 2009; Kühn and Grof, 2010). These genes are regulated by sugars, hormones, and wounding (Aoki 
et al., 1999; Meyer et al., 2004; Chen et al., 2010).

During plant growth and development, gene expression occurs in a specific order and is altered in response to changes in internal and external environmental conditions. Sugars and hormones are important signaling molecules in plants. Our previous study showed that the expression of most of the genes involved in starch synthesis was regulated by sugars and hormones in the maize endosperm (Chen et al., 2011). Starch is the major storage product in the endosperm of cereals; its synthesis is closely related to sucrose metabolism, as sucrose is an important precursor for starch biosynthesis. However, little is known regarding the transcriptional regulation of genes involved in sucrose metabolism. Thus, in this study, maize endosperms were treated with different sugars (sucrose, glucose, and fructose) and hormones [gibberellic acid (GA), indole-3-acetic acid (IAA), and abscisic acid (ABA)]. We found that sugars and hormones regulated gene expression, and most genes were regulated by ABA. Sucrose and ABA exhibited an additive effect on the expression of some genes. Differences in the transcriptional regulation of genes involved in sucrose metabolism and starch biosynthesis were observed.

\section{MATERIAL AND METHODS}

\section{Plant material growth conditions and treatment}

A maize inbred line (Mo17) was provided by the Maize Research Institute of Sichuan Agricultural University, grown at the university farm in the summer of 2013, and allowed to self-pollinate. Ten days after pollination maize endosperms were collected under aseptic conditions and approximately 20 maize endosperms were placed in MS fluid medium with different sugars ( $200 \mathrm{mM}$ sucrose, $200 \mathrm{mM}$ glucose, and $200 \mathrm{mM}$ fructose) and phytohormones $(100 \mu \mathrm{M} \mathrm{GA}, 100 \mu \mathrm{M}$ IAA, and $100 \mu \mathrm{M} \mathrm{ABA})$ at $28^{\circ} \mathrm{C}$ in the dark for $36 \mathrm{~h}$. Mannitol was added to the samples without sugars as an osmotic control (Hu et al., 2012).

\section{RNA isolation and real-time quantitative reverse transcription polymerase chain reaction (qRT-PCR) analysis}

Total RNA was extracted using Trizol reagent (Invitrogen, Carlsbad, CA, USA) and Trizol-Mate (TIANDZ, China). Reverse transcription was carried out using an RT Reagent Kit With gDNA Eraser (Perfect Real Time, Clontech, Mountain View, CA, USA). The reaction included 2 steps; the first was to remove contaminating genomic DNA and the second was reverse transcription. qRT-PCR analysis was performed using gene-specific primers and SYBR Premix Ex Taq ${ }^{\mathrm{TM}}$ II (Tli RNaseH Plus, Clontech). The qRT-PCR assays were run on a CFX96 ${ }^{\mathrm{TM}}$ Real-Time PCR system (Bio-Rad, Hercules, CA, USA). The housekeeping gene glyceraldehyde-3-phosphate-dehydrogenase (GenBank accession No.: X07156) was used for normalization in the qRT-PCR with the primers $\left(5^{\prime}-3^{\prime}\right)$ : F (ACTTCGGCATTGTTGAGG) and R (AAGTCGGTAGAAACCAGAT). The primers for genes related to sucrose metabolism are shown in Table 1. 
Table 1. Gene information and their specific primers.

\begin{tabular}{|c|c|c|c|}
\hline Enzyme/protein & Gene name & Accession No. & Primer sequence $\left(5^{\prime}-3^{\prime}\right)$ \\
\hline \multirow[t]{10}{*}{ Inv } & Ivrl & U16123 & F: GGCAGCCTCCAAACTTTCTTC \\
\hline & & & R: AACCAGTATTCTGACCGAGAGATTC \\
\hline & Ivr2 & U31451 & F: ACGAAGCTCGTCGTCCACG \\
\hline & & & R: GTTGCATTGCATCGATCAGATG \\
\hline & Incwl & U17695 & F: CCATCCACGAGGTCGAGAAG \\
\hline & & & R: CTGATAAGTTGCGATGCCTGTG \\
\hline & Incw 2 & AF050631 & F: CTGAGAGAAAAGTCGGTCACTC \\
\hline & & & R: GAAGCTCACCTCCACGTCAG \\
\hline & Incw 4 & AF043347 & F: TGCGGGGAGAAGGGCG \\
\hline & & & R: CGTCTCCGCGTGCTCAGG \\
\hline \multirow[t]{6}{*}{ SuSy } & Shl & X02400 & F: GGCTCATAACTACAAGGGCAC \\
\hline & & & R: TCCGAGTAGGGAGTGTCTTG \\
\hline & Susl & L22296 & F: TGAGCTTGTCGCCGTCTTC \\
\hline & & & R: CACCATCCTTGAGCTTCTCG \\
\hline & Sus2 & AY059416 & F: TTCCAAAACATGTCCTGCTGTATC \\
\hline & & & R: TTGATTTATGACCCGGAGC \\
\hline \multirow{2}{*}{ SPS } & ZmSPSI & M97550 & F: GCGAGAAGGGAGACACCATC \\
\hline & & & R: CACCTGTATCAGAATCACGACCTAG \\
\hline \multirow{10}{*}{ SUT } & ZmSUT1 & BAA83501 & F: GTCCGACATCGGAGCTGCTC \\
\hline & & & R: CCAGCGCCATCCAAGAACAG \\
\hline & ZmSUT2 & AAS91375 & F: TCTGTTACCCTGTACTTCGCTG \\
\hline & & & R: CTCAGTGTTGTTGTTAGCCGAC \\
\hline & ZmSUT3 & ACF86653 & F: GGTCCACTGTGCTTCACGATTC \\
\hline & & & R: GTCATGATCCGGCCTAGAAATG \\
\hline & ZmSUT5 & ACF85284 & F: ATCTCCAGGCAGGCTTGTCG \\
\hline & & & R: ATCCAAACACCTCCTTAATCGG \\
\hline & ZmSUT6 & FJ750249 & F: GAAGCTAAGGCAGACAGGGT \\
\hline & & & R: GAGGGATCCGAAAAGCTAAC \\
\hline
\end{tabular}

\section{RESULTS}

Sucrose and its decomposed products glucose and fructose, regulated by the enzymes Inv and sucrose synthase, are not only very significant metabolites but also important signaling molecule in plants, as they control the transcription and translation of numerous genes (Aoki et al., 1999; Ahn et al., 2010; Ou et al., 2013). We used different sugars to treat maize endosperm 10 days after pollination; the expression of genes involved in sucrose metabolism was evaluated using qRT-PCR. The results showed that glucose strongly stimulated the expression of Incw1, Incw4, ZmSPS1, and ZmSUT3 and slightly stimulated the expression of Ivr2, while the Ivr1, Incw 2, ZmSUT1, and ZmSUT5 mRNA was downregulated following glucose treatment (Figure 1). The transcripts of Incw 1, ZmSPS1, ZmSUT3, and ZmSUT6 were induced by fructose, but the Sus 1 and ZmSUT5 transcripts were strongly inhibited by fructose, with slight effects on the ZmSUT1 and ZmSUT2 transcripts (Figure 1). After treatment with sucrose, Ivr2, Incw 1, Incw 4, ZmSPS1, and ZmSUT3 expression were strongly upregulated, while the transcript levels of Incw2, Sus1, Sus2, ZmSUT1, ZmSUT2, and ZmSUT5 were inhibited (Figure 1).

Hormones can be synthesized by a plant, and signaling molecules affect plant growth and development by regulating gene expression (Gibson, 2004; Klingler et al., 2010; Shan et al., 2012). We used 3 hormones to treat maize endosperms and found that GA increased the abundance of ZmSUT1, ZmSUT2, and ZmSUT6 mRNA, of which the mRNA level of ZmSUT6 was most abundant. Ivr1, Incw2, and Incw 4 mRNA levels were downregulated, while there were no clear effects on the expression of other genes (Figure 1). IAA marginally stimulated Incw 1, Incw1, and ZmSUT5 expression, but distinctly inhibited Incw2 expression, while IAA 
only slightly inhibited the expression of $\operatorname{Ivr} 1$ and $S h 1$ (Figure 1). The genes tested, except for ZmSUT1, were regulated by ABA, of which only ZmSUT5 and ZmSUT6 transcripts were inhibited by $\mathrm{ABA}$ and other genes were induced by $\mathrm{ABA}$ to varying degrees; the transcripts of Incw4, Sus2, and zmSPS1 showed the highest expression (Figure1).

Previous studies showed that ABA and sucrose had an additive effect on the expression of some genes (Chen et al., 2011; Liu et al., 2011), as the effect of combined ABA and sucrose treatment were stronger compared to individual treatment with ABA or sucrose. We also found an additive effect for combined ABA and sucrose treatment on the induction of gene expression of Ivr1, Ivr2, Incw4, ZmSPS1, and ZmSUT3. However, individual treatment of ABA or sucrose may increase the abundance of $I n c w 1$ mRNA, but there was no additive effect for combined treatment with ABA and sucrose (Figure 1).

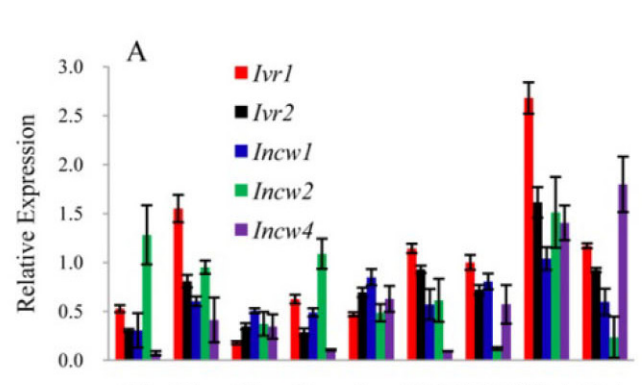

CK Man Glu Fru Suc $\mathrm{M}+\mathrm{G} \mathrm{M}+\mathrm{I} \mathrm{M}+\mathrm{A} \mathrm{S}+\mathrm{A}$

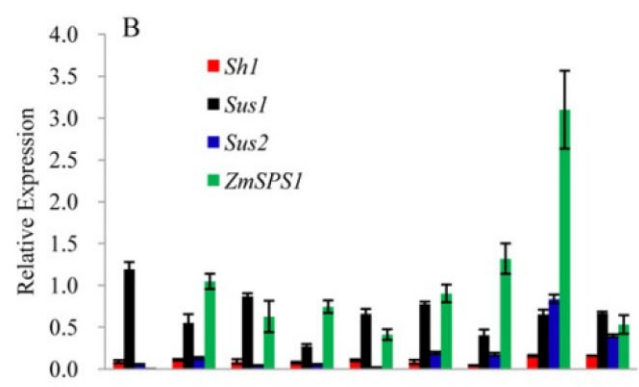

CK Man Glu Fru Suc $\mathrm{M}+\mathrm{G}$ M+I M+A S+A

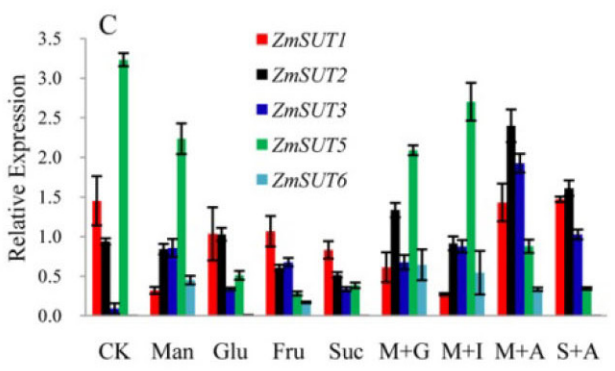

Figure 1. Ten DAP maize endosperms were separated and treated with different plant hormones and sugars [200 mM mannitol (Man), $200 \mathrm{mM}$ glucose (Glu), $200 \mathrm{mM}$ fructose (Fru), $200 \mathrm{mM}$ sucrose (Suc), $200 \mathrm{mM}$ mannitol plus $100 \mu \mathrm{M} \mathrm{GA}(\mathrm{M}+\mathrm{G}), 200 \mathrm{mM}$ mannitol plus $100 \mu \mathrm{M}$ IAA $(\mathrm{M}+\mathrm{I}), 200 \mathrm{mM}$ mannitol plus $100 \mu \mathrm{M} \mathrm{ABA}(\mathrm{M}+\mathrm{A})$, $200 \mathrm{mM}$ sucrose plus $100 \mu \mathrm{M} \mathrm{ABA}(\mathrm{S}+\mathrm{A})]$ at $28^{\circ} \mathrm{C}$ in the dark for $36 \mathrm{~h}$. Total RNA was isolated and subjected to qRT-PCR. A. Expression of Inv; B. for SuSy and SPS; C. for SUT.

\section{DISCUSSION}

Higher plants convert $\mathrm{CO}_{2}$ and $\mathrm{H}_{2} \mathrm{O}$ into carbohydrates through photosynthesis to store energy and act as the major energy source supporting plant life. Carbohydrates are also used as carbon skeletons in the synthesis of lipids, proteins, and nucleic acids (Ren and Zhang, 2013). Hormones can be synthesized by the plant, and act as signaling molecules during plant growth and development by regulating gene expression. Gene expression occurs in a specific order 
and is altered in response to changes in internal and external environmental conditions during plant growth and development. Sugars and hormones are important signaling molecules in plants in vivo, affecting numerous physiological and biochemical processes, such as flowering, stress response, seed germination, seedling growth, guard cell movement, grain filling rate, and sink strength, and among others (Yuan et al., 2006; Zhu et al., 2011; Shan et al., 2012).

Sucrose is the primary product of photosynthesis and is also the main form of longdistance transport and distribution. Moreover, sucrose and its decomposed products, glucose and fructose, are important signaling molecule in plants that regulated the expression of many genes (Aoki et al., 1999; Ahn et al., 2010; Ou et al., 2013). Maize gene expression differed with varying sugars at various concentrations. $I v r 2$ and $S u S 1$ transcripts were upregulated by increasing sugar concentration, while $\operatorname{Ivr} l$ and $S h l$ were repressed by sugars and upregulated upon sugar depletion (Koch et al., 1992; Xu et al., 1996). We found that glucose slightly induced Ivr2 mRNA and inhibited Ivrl and Sus 1 mRNA, but had no effect on Shlexpression (Figure 1). Most genes were regulated by sucrose treatment, but some were induced while others were inhibited (Figure 1). Our previous study found that genes involved in starch synthesis mainly expressed in the maize endosperm could be induced by sucrose (Chen et al., 2011). During the conversion of sucrose to starch, sucrose is not only important for starch biosynthesis but also is a regulatory molecule that modulates genes involved in the conversion of sucrose to starch. The processes of sucrose metabolism and starch biosynthesis are complex processes. In contrast, the expression of all genes tested involved in sucrose synthesis and degradation were induced by ABA (Figure 1), while the expression of genes related to starch synthesis were inhibited by ABA (Chen et al., 2011). This suggests that ABA is an important signaling molecule that strongly controls sucrose metabolism and starch biosynthesis. In maize, IAA and GA inhibited Ivr 1 mRNA, while they had little effect on Ivr2 expression (Figure 1). Ivr2 is specifically induced by water deprivation, which is related to the activity of ABA. ABA supplied directly to seedlings enhanced vacuolar Inv activity and $I v r 2$ expression (Trouverie et al., 2003, 2004). ABA may promote stomatal closure and enhance drought tolerance (Ding and Wang, 1993). Thus, Ivr2 may be involved in maize drought tolerance.

Previous studies showed that ABA and sucrose had an additive effect on the expression of some genes (Chen et al., 2011; Liu et al., 2011). Similar results were observed in our study (Figure1). However, ABA and sucrose increased the transcript levels of Incw I, but the induction of ABA combined with sucrose showed no additive effect on the expression of Incw 1 (Figure 1). This may be because the Incwlgene encodes 2 distinct transcripts, Incw I-S (small) and Incw 1-L (large), which differ in size because the 3' untranslated region and 2 transcripts induced by sucrose and glucose differ in length (Cheng et al., 1999). The specific effects of ABA on the 2 transcripts are unknown. We found that total Incwl mRNA was increased following treatment with ABA (Figure 1). The conversion of sucrose to starch was tightly regulated by signaling molecules. However, the signal transduction pathway (perception, recognition, and transmission) remains unclear. Little information regarding sugars or hormones responsive to cis-acting elements has been reported. Additionally, there have been few studies examining transcription factors involved in regulating signal transduction. Starch is a major storage product in the endosperm of cereals, and its synthesis is closely related to sucrose metabolism. Further studies examining the mechanisms of regulating the expression of genes involved in sucrose metabolism and starch biosynthesis should be conducted to further understand the mechanism of the sucrose to starch conversion. 


\section{ACKNOWLEDGMENTS}

Research supported by the National Key Basic Research Program of China (\#2014CB138200), Cultivating Fund of Excellent Master's Degree Theses of Sichuan Agriculture University, and the Preferentially Financing Projects of Scientific and Technological Activities of Overseas Students in Sichuan Province.

\section{REFERENCES}

Ahn YO, Kim SH, Kim CY, Lee JS, et al. (2010). Exogenous sucrose utilization and starch biosynthesis among sweet potato cultivars. Carbohydr. Res. 345: 55-60.

Aoki N, Hirose T, Takahashi S, Ono K, et al. (1999). Molecular cloning and expression analysis of a gene for a sucrose transporter in maize (Zea mays L.). Plant Cell Physiol. 40: 1072-1078.

Carlson SJ, Chourey PS, Helentjaris T and Datta R (2002). Gene expression studies on developing kernels of maize sucrose synthase (SuSy) mutants show evidence for a third SuSy gene. Plant Mol. Biol. 49: 15-29.

Chen JY, Liu SL, Siao W and Wang SJ (2010). Hormone and sugar effects on rice sucrose transporter OsSUT1 expression in germinating embryos. Acta Physiol. Plant 32: 749-756.

Chen J, Huang B, Li Y, Du H, et al. (2011). Synergistic influence of sucrose and abscisic acid on the genes involved in starch synthesis in maize endosperm. Carbohydr. Res. 346: 1684-1691.

Cheng WH, Taliercio EW and Chourey PS (1996). The miniature1 seed locus of maize encodes a cell wall invertase required for normal development of endosperm and maternal cells in the pedicel. Plant Cell 8: 971-983.

Cheng WH, Taliercio EW and Chourey PS (1999). Sugars modulate an unusual mode of control of the cell-wall invertase gene (Incw1) through its 3' untranslated region in a cell suspension culture of maize. Proc. Natl. Acad. Sci. U. S. A. 96: 10512-10517.

Chourey PS, Jain M, Li QB and Carlson SJ (2006). Genetic control of cell wall invertases in developing endosperm of maize. Planta 223: 159-167.

Cui XH, Murthy HN, Wu CH and Paek KY (2010). Sucrose-induced osmotic stress affects biomass, metabolite, and antioxidant levels in root suspension cultures of Hypericum perforatum L. Plant Cell Tiss. Organ. Cult. 103: 7-14.

Ding L and Wang XC (1993). The role of abscisic acid in stomatal responses to drought stress. Agr. Res. Arid. Areas 11: $50-56$.

Gibson SI (2004). Sugar and phytohormone response pathways: navigating a signalling network. J. Exp. Bot. 55: 253-264.

Gupta AK and Kaur N (2005). Sugar signalling and gene expression in relation to carbohydrate metabolism under abiotic stresses in plants. J. Biosci. 30: 761-776.

Gupta M, Chourey PS, Burr B and Still PE (1988). cDNAs of two non-allelic sucrose synthase genes in maize: cloning, expression, characterization and molecular mapping of the sucrose synthase-2 gene. Plant Mol. Biol. 10: 215-224.

Hu YF, Li YP, Zhang J, Liu H, et al. (2012). Binding of ABI4 to a CACCG motif mediates the ABA-induced expression of the ZmSSI gene in maize (Zea mays L.) endosperm. J. Exp. Bot. 63: 5979-5989.

Kim JY, Mahé A, Brangeon J and Prioul JL (2000a). A maize vacuolar invertase, IVR2, is induced by water stress. Organ/ tissue specificity and diurnal modulation of expression. Plant Physiol. 124: 71-84.

Kim JY, Mahé A, Guy S, Brangeon J, et al. (2000b). Characterization of two members of the maize gene family, Incw3 and Incw4, encoding cell-wall invertases. Gene 245: 89-102.

Klingler JP, Batelli G and Zhu JK (2010). ABA receptors: the START of a new paradigm in phytohormone signalling. $J$. Exp. Bot. 61: 3199-3210.

Koch KE, Nolte KD, Duke ER, McCarty DR, et al. (1992). Sugar levels modulate differential expression of maize sucrose synthase genes. Plant Cell 4: 59-69.

Kühn C and Grof CP (2010). Sucrose transporters of higher plants. Curr Opin. Plant Biol 13: 288-298.

Liu YH, Qin JY, Huang XZ and Hu YF (2011). Effects of exogenous hormones and carbohydrates on expression of zSs1 in maize. J. Nucl. Agric. Sci. 25: 432-435.

Lutfiyya LL, Xu N, D’Ordine RL, Morrell JA, et al. (2007). Phylogenetic and expression analysis of sucrose phosphate synthase isozymes in plants. J. Plant Physiol. 164: 923-933.

Ma XL, Liu YH, Yuan ZL and Shi YS (2009). Cloning of cDNAs for a novel sugar transporter gene, ZmERD6, from maize and its expression analysis under abiotic stresses. Acta Agron. Sin. 35: 1410-1417.

McElfresh KC and Chourey PS (1988). Anaerobiosis induces transcription but not translation of sucrose synthase in maize. Plant Physiol. 87: 542-546. 
Meyer S, Lauterbach C, Niedermeier M, Barth I, et al. (2004). Wounding enhances expression of AtSUC3, a sucrose transporter from Arabidopsis sieve elements and sink tissues. Plant Physiol. 134: 684-693.

Ou Y, Song B, Liu X, Xie C, et al. (2013). Promoter regions of potato vacuolar invertase gene in response to sugars and hormones. Plant Physiol. Biochem. 69: 9-16.

Ren X and Zhang J (2013). Research progresses on the key enzymes involved in sucrose metabolism in maize. Carbohydr. Res. 368: 29-34.

Rowland LJ, Chen YC and Chourey PS (1989). Anaerobic treatment alters the cell specific expression of Adh-1, Sh, and Sus genes in roots of maize seedlings. Mol. Gen. Genet. 218: 33-40.

Shan X, Yan J and Xie D (2012). Comparison of phytohormone signaling mechanisms. Curr. Opin. Plant Biol. 15: 84-91.

Shanker S, Salazar RW, Taliercio EW and Chourey PS (1995). Cloning and characterization of full-length cDNA encoding cell-wall invertase from maize. Plant Physiol. 108: 873-874.

Shin DH, Choi MG, Lee HK, Cho M, et al. (2013). Calcium dependent sucrose uptake links sugar signaling to anthocyanin biosynthesis in Arabidopsis. Biochem. Biophys. Res. Commun. 430: 634-639.

Tognetti JA, Pontis HG and Martinez-Noel GM (2013). Sucrose signaling in plants: a world yet to be explored. Plant Signal. Behav. 8: e23316.

Trouverie J, Thevenot C, Rocher JP, Sotta B, et al. (2003). The role of abscisic acid in the response of a specific vacuolar invertase to water stress in the adult maize leaf. J. Exp. Bot. 54: 2177-2186.

Trouverie J, Chateau-Joubert S, Thevenot C, Jacquemot MP, et al. (2004). Regulation of vacuolar invertase by abscisic acid or glucose in leaves and roots from maize plantlets. Planta 219: 894-905.

Werr W, Frommer WB, Maas C and Starlinger P (1985). Structure of the sucrose synthase gene on chromosome 9 of Zea mays L. EMBO J. 4: 1373-1380.

Xu J, Pemberton GH, Almira EC, McCarty DR, et al. (1995). The Ivr 1 gene for invertase in maize. Plant Physiol. 108: 1293-1294.

Xu J, Avigne WT, McCarty DR and Koch KE (1996). A similar dichotomy of sugar modulation and developmental expression affects both paths of sucrose metabolism: evidence from a maize Invertase gene family. Plant Cell 8: 1209-1220.

Yuan K and Wysocka-Diller J (2006). Phytohormone signalling pathways interact with sugars during seed germination and seedling development. J. Exp. Bot. 57: 3359-3367.

Zeng Y, Wu Y, Avigne WT and Koch KE (1998). Differential regulation of sugar-sensitive sucrose synthases by hypoxia and anoxia indicate complementary transcriptional and posttranscriptional responses. Plant Physiol. 116: 1573-1583.

Zeng Y, Wu Y, Avigne WT and Koch KE (1999). Rapid repression of maize invertases by low oxygen. Invertase/sucrose synthase balance, sugar signaling potential, and seedling survival. Plant Physiol. 121: 599-608.

Zhu G, Ye N, Yang J, Peng X, et al. (2011). Regulation of expression of starch synthesis genes by ethylene and ABA in relation to the development of rice inferior and superior spikelets. J. Exp. Bot. 62: 3907-3916. 